\title{
MINI PERCUTANEOUS NEPHROLITHOTOMY VERSUS EXTRACORPOREAL SHOCK WAVE LITHOTRIPSY IN TREATMENT OF NON LOWER POLAR HIGH DENSITY RENAL STONE 10-20 MM
}

\section{(A PROSPECTIVE RANDOMIZED STUDY)}

\author{
By \\ Mahmoud A. El-Mesery, Abul-fotouh A. Abul-fotouh and Hassan A. \\ Hassan
}

Department of Urology, Faculty of Medicine, Al Azhar University (Cairo)

Corresponding author: Mahmoud A. El-Mesery,

Mobile: 0109735376, E-mail: adelelmesery1@ gmail.com

\begin{abstract}
Background: The optimal management of medium sized renal stones differs from one patient to another according to different factors. Stone attenuation value (SAV) is one of these factors which affect stone free rate (SFR) after extracorporeal shock wave lithotripsy (ESWL) with limited effect on mini-percutaneous nephrolithotomy (mini-PCNL).

Objective: To compare safety and SFR of mini-PCNL and ESWL in the treatment of non-lower pole renal stones with high density and size of 10-20 mm.

Patients and Methods: This prospective randomized study was carried out at Sayed Galal, Al Azhar University Hospital, Cairo, Egypt from November 2019 to October 2020 and included 70 patients with nonlower pole renal calculi 10 to $20 \mathrm{~mm}$. Patients were randomly allocated in 2 equal groups: Group A was treated by mini-PCNL and group B was treated by ESWL. The primary end point was SFRs (no stone or residual 3>mm) in ESWL and mini-PCNL in this category of stone. The secondary end points were the complication rate, retreatment rate, and need for auxiliary procedures in each group.
\end{abstract}

Results: The overall operative time was significantly lower in mini-PCNL group (median: 50.00; IQR: 20.00) compared to ESWL group (median: 55.00; IQR: 28.00) $(\mathrm{p}=0.001)$. The overall fluoroscopy time was significantly lower in mini-PCNL than ESWL (3.2 versus 3.6 minutes, p=0.040). In mini-PCNL group, 32 out of $34(94.1 \%)$ patients were stone free. In ESWL group, 10 out of $33(30.1 \%)$ were rendered stone free after the third ESWL session. The SFR was significantly higher in mini-PCNL group ( $<<0.001)$. In miniPCNL group, none of cases needed a second look PCNL. The 2 failed cases had significant residual fragments that migrated into inaccessible calyx during pneumatic lithotripsy. In ESWL group, all cases had normal hemoglobin $(\mathrm{Hb})$ level at each follow-up visit. In mini-PCNL group, when comparing pre- and postoperative $\mathrm{Hb}$, a very highly significant differences were observed between pre- and post-operative Hb level $(\mathrm{p}<0.001)$.

Conclusions: Mini-PCNL is superior to ESWL in treatment of non-lower pole medium sized renal stones of high density with high SFR, and low complication rate as need for re-hospitalization or need for auxiliary procedure.

Keywords: Percutaneous Nephrolithotomy, Extracorporeal Shock Wave Lithotripsy, Stone attenuation value, Stone free rate, Non-lower pole, renal stones. 


\section{INTRODUCTION}

The European Association of Urology (EAU) Guidelines of urolithiasis stated that the treatment options for renal stones 10-20 mm include either Extracorporeal shock wave lithotripsy (ESWL) or endourology (retrograde intrarenal surgery (RIRS) and percutaneous nephrolithotomy (PCNL) (Turk et al., 2016).

However, the limitations of ESWL include relatively lower stone free rate (SFR) and the need for repeated sessions and auxiliary procedures especially in lower pole and harder stones. In a study divided the patients in to 3 groups according to stone attenuation value (SAV) : <500, 500-1000, and >1000 Hounsfield units (HU), SFR was $100 \%$, $95.7 \%$, and $44.6 \%$ respectively. Also, the number of sessions increases with increased SAV. SFR after the first session in these 3 groups was $100 \%, 49 \%$, and $0 \%$ respectively (Massoud et al., 2014).

In another study, SFR in patients with a stone diameter less than $10 \mathrm{~mm}$ was $79.3 \%$ and $45.7 \%$ in those with stone diameter above $10 \mathrm{~mm}$. Regarding SAV, SFR was $93.8 \%, 62.7 \%$, and $24.5 \%$ in the groups with SAV $<500 \mathrm{HU}, 500$ to 1,000 $\mathrm{HU}$, and $>1,000 \mathrm{HU}$, respectively (Waqas et al., 2018).

PCNL is now considered the 'gold standard' treatment for managing simple and complex renal stones, with a success rate of $>90 \%$ (Gonen and Basaran, 2014; Ferakis and Stavropoulos, 2015). It has good SFR but is associated with a significant risk of morbidity (Zeng et al, 2013).

Most of the complications associated with PCNL are related to the tract size.
So, reduction in tract size can lower the complications associated with it (Yamaguchi et al., 2011; Mishra et al., 2011).

The term mini-PCNL is usually used for smaller diameter sheaths (12 to $18 \mathrm{~F}$ compared to 24-30 F in standard PCNL). It has lower morbidity due to less bleeding, and reduced pain (Ferakis and Stavropoulos, 2015).

Mini-PCNL has comparable SFR to the standard method, even for large stones. In a study comparing mini-PCNL and standard PCNL, the clearance rates were $96 \%$ and $100 \%$, respectively at 1 month follow up (Mishra et al., 2011).

The European guidelines put ESWL and PCNL as an equal treatment options for non-lower pole renal stones from 10 to $20 \mathrm{~mm}$ size regardless SAV. But, there are several studies reporting markedly reduced SFR after ESWL with increased SAV. Several studies were comparing ESWL with PCNL or RIRS in lower calyceal stones, but no studies to compare these options in non-lower pole stones with high density.

The present work aimed to compare safety and SFR of mini-PCNL and ESWL in the treatment of non-lower pole renal stones with high density and size of 10-20 $\mathrm{mm}$.

\section{PATIENTS AND METHODS}

This study was prospective randomized study comparing mini-PCNL versus ESWL in treating non-lower pole high dense renal stones of $10-20 \mathrm{~mm}$ size. The study was conducted at the Department of Urology, Sayed Galal University Hospital, Cairo, Egypt, The study was done 
between November, 2019 and October, 2020. Inclusion criteria were single nonlower pole medium size $(10-20 \mathrm{~mm})$ and high dense (> $1000 \mathrm{HU}$ ) renal stones. Patients with BMI > $40 \mathrm{~kg} / \mathrm{m} 2$, uncorrected bleeding disorders, solitary kidney, anatomical renal abnormalities, obstruction distal to the stone or those below 18 years were excluded.

Patients were evaluated preoperatively through history, physical examination, BMI, urine analysis with culture and sensitivity, $\mathrm{CBC}$, Serum creatinine, $\mathrm{Hb}$, plain urinary tract (PUT), computed tomography (CT), pelviabdominal ultrasonography (US). Intraoperative evaluation of fluoroscopy time, operative time and complications such as bleeding, perforation, hypotension and organ injury was done. Postoperative evaluation of $\mathrm{Hb}$, creatinine, SFR and complications as fever, hematuria, leakage and urosepsis was done.

In group (A), the procedure was carried out under general anesthesia. In lithotomy position, a $6 \mathrm{Fr}$ ureteral catheter was introduced through cystoscopy. Then patient was put in the prone position. Under fluoroscopic guidance and after opacification of the pelvicaliceal system with contrast, the desired calyx was punctured using 18-G puncture needle, followed by insertion of guide wire (0.035inch, J-tip). Another guide wire was inserted as a safety wire. Tract dilation was performed up to $16 \mathrm{~F}$ sheath using either Alkan or Amplatz dilators. Miniature nephroscope 12 Fr Karl Storz was used, stone fragmentation was done by pneumatic lithotripsy. The fragments were removed using stone forceps or Zero Tip baskets. The collecting system was examined by endoscopically and by fluoroscopy to confirm complete stone clearance. At the end of procedure, 14-F nephrostomy tube was inserted and nephrostogram was done to exclude any significant extravasation or perforation. Then nephrostomy tube was fixed to the skin and closed. Ureteric catheter was left in place. Nephrostomy tube was placed for $24 \mathrm{~h}$ then removed on postoperative day 1 , the ureteric catheter was removed on postoperative day 2 then patient was discharged. In group (B), all patients were treated by Dornier lithotripter SII. All patients were treated in supine position with the water cushion adjusted below the flank. The localization was done by fluoroscopy. A maximum of 3000 shocks was given at each session or until complete fragmentation occurs at a rate of 80 shocks per minute and maximum power 3-4. Group A (mini-PCNL): Patients were considered to be stone free if there was no stone on US or PUT or stone $<3 \mathrm{~mm}$. Group B (ESWL): Patients were considered to be stone free if there was no stone on US or PUT or stone $<3$ $\mathrm{mm}$ after two weeks of last sitting. The primary end points were the SFRs in ESWL and mini-PCNL in this category of stone. The secondary end points were the complication rate, retreatment rate, and need for auxiliary procedures in each group.

\section{Statistical analysis:}

Statistical analysis was performed using SPSS 17.0 for Windows software (SPSS, Inc, Chicago, IL, USA) with the Student's $t$ test for continuous variables and the chi-square and Fisher's exact tests for categorical variables. The continuous variables were compared between groups 
using independent sample t-test or MannWitney U test. Pre-operative and postoperative $\mathrm{Hb}$ levels were compared using Wilcoxon signed-rank test. Differences resulting in a $\mathrm{P}$ value of $<0.05$ were considered statistically significant. The data was presented in the form of percentage, tables and numbers.

\section{RESULTS}

The overall operative time was significantly lower in mini-PCNL group (median: 50.00; IQR: 20.00) compared to ESWL group (median: 55.00; IQR: 28.00) $(p=0.001)$. The overall fluoroscopy time was significantly lower in mini-PCNL than ESWL (3.2 versus 3.6 minutes, $\mathrm{p}=0.040$ ). In mini-PCNL group, 32 out of $34(94.1 \%)$ patients were stone free. In ESWL group, 10 out of $33(30.1 \%)$ were rendered stone free after the third ESWL session. The SFR was significantly higher in mini-PCNL group $(\mathrm{p}<0.001)$ (Figure 1).

In mini-PCNL group, none of cases needed a second look PCNL. The 2 failed cases had significant residual fragments that migrated into inaccessible calyx during pneumatic lithotripsy. One of them refused further intervention and the second was referred for ESWL unit. No data available about the result of ESWL in this patient.

In ESWL group, none of cases became stone free after the first ESWL session. One case became stone free after the second session, and nine after the third session. Two of failed cases underwent ureteroscopy (URS) after the second ESWL session; one due to steinstrasse and one due to downward migration of unfragmented stone upper calyx into the lower ureter. Eight of failed cases were shifted for mini-PCNL during the study period and all osf them became stone free (data not included) (Figure 2).

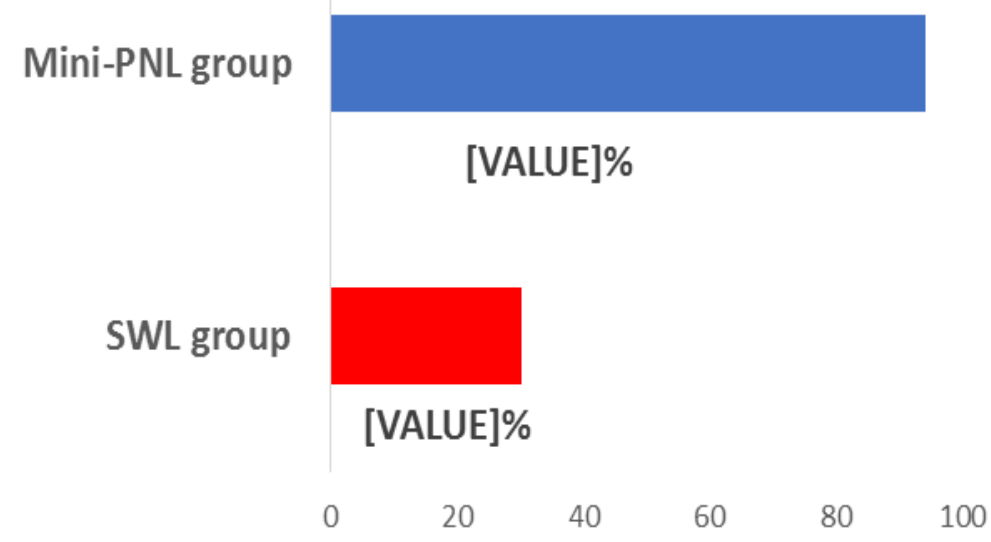

Figure (1): Bar chart demonstrating the stone free rate in both groups. 


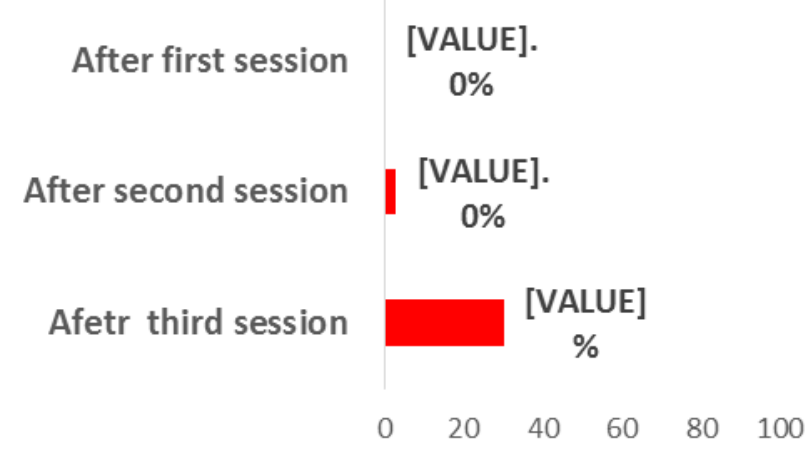

Figure (2): Bar chart demonstrating the cumulative stone free rate in ESWL group after each ESWL session

The median pre-operative $\mathrm{Hb}$ level was $13.70 \mathrm{gm} / \mathrm{dl}$ (IQR:0.90), there was no significant differences observed between mini-PCNL and ESWL groups regarding the preoperative $\mathrm{Hb}$. In ESWL group, all cases had normal $\mathrm{Hb}$ level at each followup visit. In mini-PCNL group, significant differences were observed between pre and post-operative $\mathrm{Hb} \quad(\mathrm{p}<0.001)$. The median reduction of $\mathrm{Hb}$ was $1.00 \mathrm{gm} / \mathrm{dL}$ (IQR: 2.15). In mini-PCNL group, only one case required post-operative blood transfusion. The main indication of blood transfusion was reduction of postoperative $\mathrm{Hb} \quad(<7$ gm/dL). In ESWL group, all sessions were performed as a day-case procedure. In mini-PCNL group, the median hospitalization time was 4 days (IQR:0.00).

Regarding unscheduled rehospitalization, 5 cases (all in ESWL group) were re-hospitalized; 3 of them due to persistent renal pain, one due to gross hematuria and one due to acute pyelonephritis. The unscheduled rehospitalization rate was significantly higher in ESWL group (15.2\% vs. 0.0\%; $\mathrm{p}=0.018$ ).

In mini-PCNL group, 8 cases developed peri-operative complication. Perforation of renal pelvis with intraoperative bleeding was reported in one case. Post-operative complications were reported in 7 cases. The most common post-operative complication was fever $(15.3 \%)$ followed by transient urine leakage after nephrostomy tube removal $(10.6 \%)$. The intra- and post-operative complications, its modified clavien classification (MCC) grades and management were summarized in Table (1). 
Table (1): Intra- and post-operative complications in mini-PCNL group

\begin{tabular}{|c|c|c|c|}
\hline & $\begin{array}{l}\text { MCC } \\
\text { Grade }\end{array}$ & $\begin{array}{c}\text { Number } \\
(34)\end{array}$ & $\%$ \\
\hline Overall & & 8 & 23.5 \\
\hline Intra-operative: & & 1 & 2.9 \\
\hline - Bleeding requiring blood transfusion & 2 & 1 & 2.9 \\
\hline \multicolumn{4}{|c|}{ Management: transfusion of one unit of whole blood + IV crystalloid infusion } \\
\hline - Perforation of renal pelvis & $3 b$ & 1 & 2.9 \\
\hline \multicolumn{4}{|c|}{ Management: double-J ureteral stenting (no long-term follow-up data) } \\
\hline Post-operative: & & 7 & 20.6 \\
\hline - Post-operative fever & 2 & 4 & 11.8 \\
\hline \multicolumn{4}{|l|}{ Management: IV broad-spectrum antibiotics } \\
\hline - Transient bleeding & 1 & 2 & 5.9 \\
\hline \multicolumn{4}{|c|}{ Management: clamping of NP tube + IV crystalloid infusion } \\
\hline $\begin{array}{l}\text { - Transient urine leakage after removal of NP } \\
\text { tube }\end{array}$ & 1 & 3 & 8.8 \\
\hline
\end{tabular}

IV, Intravenous; MCC, Modified Clavian classification; PCNL, Percutaneous nephrolithotomy; NP, Nephrostomy.

The total number of complications is more than the actual number of cases as some patients had more than one complication. In ESWL group ,no reported intra-procedural complications. Five cases developed post-procedural complications and all of them needed hospital admission. Two cases had persistent renal pain and imaging study revealed steinstrasse (after the 2nd ESWL session) in one and stone migration down from upper calyx into the lower ureter (after the 1st ESWL session) in the other. The first case underwent double-J ureteral stent fixation. The second case underwent URS with stone fragmentation and extraction (data not included). Postoperative complications, its MCC grades and management are summarized in Table (2).

Table (2): Post-procedure complications in ESWL group

\begin{tabular}{|c|c|c|c|}
\hline & $\begin{array}{l}\text { MCC } \\
\text { Grade }\end{array}$ & $\begin{array}{c}\text { Number } \\
(33)\end{array}$ & $\%$ \\
\hline Overall & & 5 & 15.2 \\
\hline - Renal pain & 1 & 1 & 3.0 \\
\hline \multicolumn{4}{|l|}{ Management: analgesics } \\
\hline - Hematuria & 1 & 1 & 3.0 \\
\hline \multicolumn{4}{|l|}{ Management: conservative } \\
\hline - Steinstrasse & $3 b$ & 1 & 3.0 \\
\hline \multicolumn{4}{|c|}{ Management: URS and double-J ureteral stent placement } \\
\hline - Migration of stone into lower ureter & $3 \mathrm{~b}$ & 1 & 3.0 \\
\hline \multicolumn{4}{|c|}{ Management: URS, and stone fragmentation and extraction } \\
\hline - Pyelonephritis & 2 & 1 & 3.0 \\
\hline
\end{tabular}

IV, Intravenous; MCC, Modified Clavian classification; ESWL, Shockwave lithotripsy; URS, ureteroscopy. 
The total number of complications is more than the actual number of cases as some patients had more than one complication. On comparing the overall complication rates, no significant difference was observed between miniPCNL and ESWL groups $(23.5 \%$ vs. $15.2 \% ; \mathrm{p}=386$ ). of lower calyceal stones, ESWL has high SFRs for renal stones $20 \mathrm{~mm}$ or less (Zheng et al. 2015). SFRs after single treatment for medium sized stones $10-30 \mathrm{~mm}$ are $95.3 \%$ for PCNL vs $87.8 \%$ for RIRS and $60.4 \%$ for ESWL

\section{DISCUSSION}

The ideal treatment for the stone 10$20 \mathrm{~mm}$, non-lower pole, with density above $1000 \mathrm{HU}$ is that provide the highest SFRs with fewer sessions, and minimal complications. In this study, mini-PCNL provided higher SFRs $(97.1 \%)$ than ESWL (30.3\%). Also, in mini-PCNL, all patients in the success group were stone free after one procedure. While, in ESWL group, none of the patients became stonefree after the first session, and this SFR was achieved after 3 sessions. Our results showed high discrepancy between the SFRs in both groups.

In our series, $\mathrm{Hb}$ drop was highly significant in mini-PCNL group in comparison with ESWL. The mean $\mathrm{Hb}$ drop was $1.2 \mathrm{~g} / \mathrm{dL}$.

There were no reported intraoperative complications in ESWL group. While in mini-PCNL, intraoperative complications were recorded only in one patient. There was no significant difference regarding postoperative complications in both groups.

The mean operative time and fluoroscopy time were significantly lower
(Wiesenthal et al., 2011). In a study done by Chung and Turney on the efficacy of ESWL on medium sized renal stones 10$20 \mathrm{~mm}$, the overall SFR was $66.4 \%$. The results were similar in different stone locations. But, SFRs were higher $(70.4 \%)$ with stone size $<15 \mathrm{~mm}$ compared to only $53.1 \%$ in stone size $15-20 \mathrm{~mm}$ (Chung and Turney, 2016). In spite of achieving high SFRs with PCNL, it is also associated with increased risk of bleeding and blood transfusion (Yamaguchi et al, 2011).,

in the mini-PCNL group compared with the ESWL group (51 versus 63 minutes, and 3.2 versus 3.6 minutes, respectively).

In several studies, the overall SFRs after ESWL for medium sized renal stones were $66.4 \%$ (Chung and Turney, 2016), $75 \%$ (Hassan et al, 2015), $79.2 \%$ (Wiesenthal et al, 2011), and $83 \%$ Massoud et al. 2014). With the exception of lower calyceal stones, ESWL has high SFRs for renal stones $20 \mathrm{~mm}$ or less (Zheng et al. 2015). SFRs after single treatment for medium sized stones 10$30 \mathrm{~mm}$ are $95.3 \%$ for PCNL vs $87.8 \%$ for RIRS and $60.4 \%$ for ESWL (Wiesenthal et al., 2011). In a study done by Chung and Turney on the efficacy of ESWL on medium sized renal stones $10-20 \mathrm{~mm}$, the overall SFR was $66.4 \%$. The results were similar in different stone locations. But, SFRs were higher $(70.4 \%)$ with stone size $<15 \mathrm{~mm}$ compared to only $53.1 \%$ in stone size 15-20 mm (Chung and Turney, 2016). In spite of achieving high SFRs with PCNL, it is also associated with increased risk of bleeding and blood transfusion (Yamaguchi et al, 2011)., 
As regard $\mathrm{Hb}$ drop, similar results were reported in a study comparing RIRS with mini-PCNL in multiple renal stones 10$30 \mathrm{~mm}$, the $\mathrm{Hb}$ drop was high in miniPCNL group and was $1 \mathrm{gm} / \mathrm{dL}$ (Yanaral et al., 2019). But, this Hb drop is lower than that in standard PCNL. In a study comparing standard and mini-PCNL, the overall SFRs were high in both groups, $97.1 \%$ and $95.4 \%$. The $\mathrm{Hb}$ drop was significantly lower in mini-PCNL group The mean $\mathrm{Hb}$ drop and the bleeding necessitating blood transfusion were 0.6 vs $1.9 \mathrm{gm}$ and $1.2 \mathrm{vs} 9.8 \%$ in miniPCNL and standard PCNL groups, respectively (Sakr et al., 2017).

Also, in a study comparing mini-PCNL and standard PCNL, the mean operation time in mini-PCNL was 83 minutes, and mean fluoroscopy time was 4.3 minutes (Sakr et al, 2017). The postoperative hospitalization time was significantly higher in the mini-PCNL group But, unscheduled hospital readmission was reported only in ESWL group in about $15 \%$ Similarly, in a study comparing ESWL, RIRS, and PCNL in treatment of medium sized renal stones, postoperative hospitalization was significant in PCNL group (Wiesenthal et al., 2011).

\section{CONCLUSION}

Mini-PCNL is superior to ESWL in treatment of non-lower pole medium sized renal stones of high density with high SFR, and low complication rate as need for re-hospitalization or need for auxiliary procedure.

\section{REFERENCES}

1. Chung VY and Turney BW (2016): The success of shock wave lithotripsy (ESWL) in treating moderate-sized $(10-20 \mathrm{~mm})$ renal stones. Urolithiasis, 44(5): 441-444.

2. Ferakis $N$ and Stavropoulos $M$ (2015): Mini percutaneous nephrolithotomy in the treatment of renal and upper ureteral stones: lessons learned from a review of the literature. Urol Ann., 7:141-148.

3. Gonen $M$ and Basaran $B$ (2014): Tubeless percutaneous nephrolithotomy: spinal versus general anesthesia. Urol J., 11:1211-1215.

4. Hassan, M., El-Nahas, A. R., Sheir, K. Z., El-Tabey, N. A., El-Assmy, A. M., Elshal, A. M., \& Shokeir, A. A. (2015): Percutaneous nephrolithotomy vs. extracorporeal shockwave lithotripsy for treating a $20-30 \mathrm{~mm}$ single renal pelvic stone. Arab journal of urology, 13(3), 212216.

5. Massoud, A. M., Abdelbary, A. M., AlDessoukey, A. A., Moussa, A. S., Zayed, A. S., \& Mahmmoud, O. (2014): The success of extracorporeal shock-wave lithotripsy based on the stone-attenuation value from non-contrast computed tomography. Arab Journal of Urology, 12(2), 155-161.

6. Mishra, S., Sharma, R., Garg, C., Kurien, A., Sabnis, R., \& Desai, M. (2011): Prospective comparative study of miniperc and standard PNL for treatment of 1 to $2 \mathrm{~cm}$ size renal stone. BJU international, 108(6), 896-900.

7. Sakr, A., Salem, E., Kamel, M., Desoky, E., Ragab, A., Omran \& Shahin, A. (2017): Minimally invasive percutaneous nephrolithotomy vs standard PCNL for management of renal stones in the flankfree modified supine position: singlecenter experience. Urolithiasis, 45(6), 585589.

8. Türk, C., Petrŕ́k, A., Sarica, K., Seitz, C., Skolarikos, A., Straub, M., \& Knoll, T. (2016): EAU guidelines on interventional 
treatment for urolithiasis. European urology, 69(3), 475-482.

9. Waqas M., Imran Jamil, M., \& Akhrer ,S. (2018): Evaluating the importance of different computed tomography scan-based factors in predicting the outcome of extracorporeal shock wave lithotripsy for renal stones, Investig Clin Urol., 59(1): 25-31.

10.Wiesenthal J. D., Ghiculete, D., Honey, R. J. D. A., \& Pace, K. T. (2011): A comparison of treatment modalities for renal calculi between 100 and $300 \mathrm{~mm} 2$ : are shockwave lithotripsy, ureteroscopy, and percutaneous nephrolithotomy equivalent? J Endourol., 25(3): 481-485.

11.Yamaguchi A., Skolarikos A., Buchholz N. P. N., Chomon, G. B., Grasso, M., Saba, P. \& De la Rosette, on behalf of the clinical Research office of the Endourological society of Percutaneous Nephrolithotomy Study Group, J. (2011): Operating times and bleeding complications in percutaneous nephrolithotomy: a comparison of tract dilation methods in 5537 patients in the Clinical Research Office of the Endourological Society Percutaneous Nephrolithotomy Global Study. J Endourol., 25(6): 933-939.

12.Yanaral F., Ozgor F., Kucuktopcu O., Sarilar O., Ayranci A., Savun M., (2019): Comparison of Flexible Ureterorenoscopy and Mini Percutaneous Nephrolithotomy in the Management of Multiple Renal Calculi in 10-30 mm Size. Urol J, 16(4): 326-330.

13.Zeng, G., Zhao, Z., Wan, S., Mai, Z., Wu, W., Zhong, W., \& Yuan, J. (2013): Minimally invasive percutaneous nephrolithotomy for simple and complex renal caliceal stones: a comparative analysis of more than 10,000 cases. Journal of endourology, 27(10),1203-1208

14.Zheng C., Yang H., Luo J., Xiong B., Wang H., \& Jiang Q., (2015): Extracorporeal shock wave lithotripsy versus retrograde intrarenal surgery for treatment for renal stones $1-2 \mathrm{~cm}$ : a metaanalysis. Urolithiasis, 43(6): 549-556. 


\section{مقارنة إستخراج الحصوات بمنظار الكلى الصفير عن طريق الجلد وتثتيت الحصوات بالموجات المسلمية من خارج الجسم فى علاج حصوات الكلى فى غير الكأس السفلى وذات الكثافة العالية وحجم مز 10 الى $\infty 20$}

\section{(دراسة مسثقبلية مشوائية)}

محمود عادل المسيري، أبوالفتوح عبدالمجيد أبوالفتوح، حسن عبد العظيم حسن

قسم جراحة المسالك البولية، كلية الطب، جامعة الأزهر (القاهرة)

E-mail: adelelmesery1@gmail.com

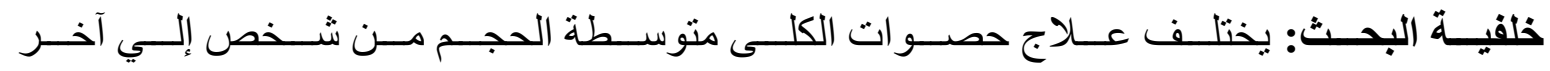

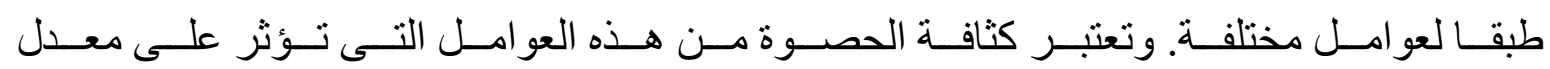

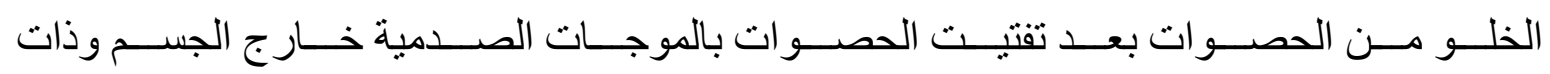
تأثير بسيط علي إستخر اج الحصوات بمنظار الكلى الصغير.

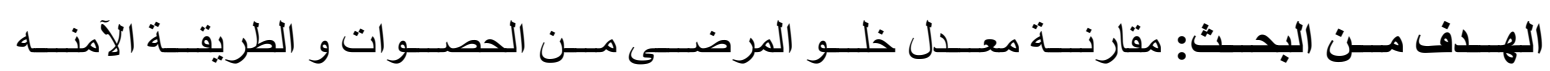

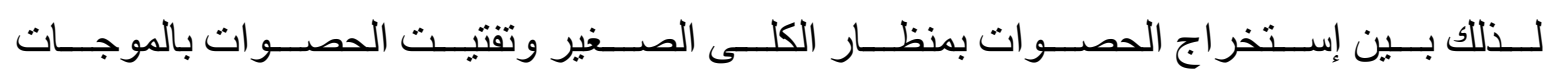
الصدمية وذلك فى الحصوات ذات الكثافة العالية ومتوسطة الحجم.

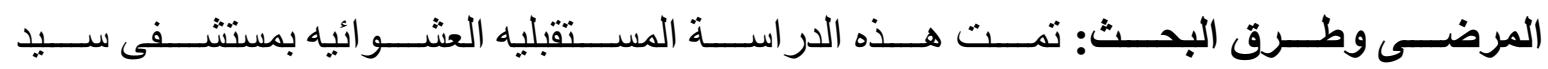

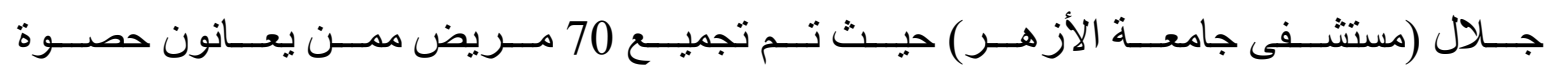

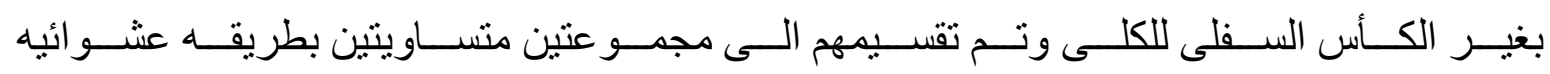

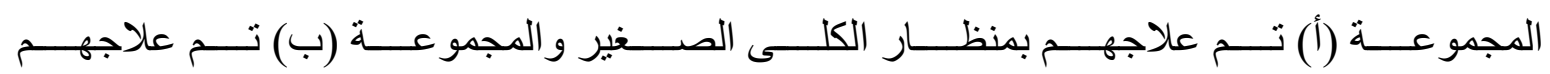
بتفنيت الحصوات بالموجات الصدمية.

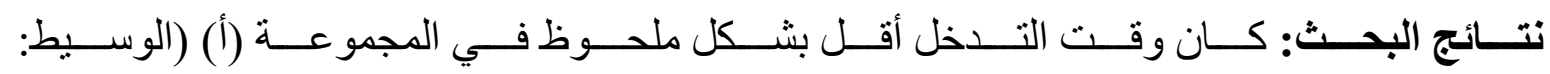

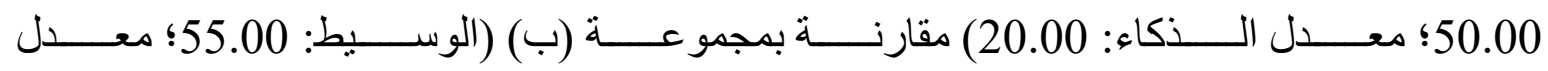

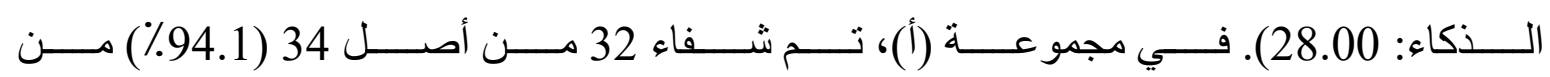

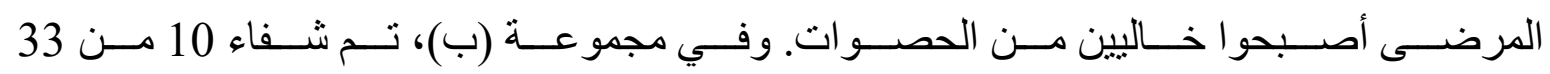

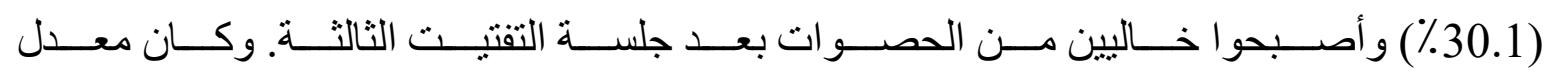




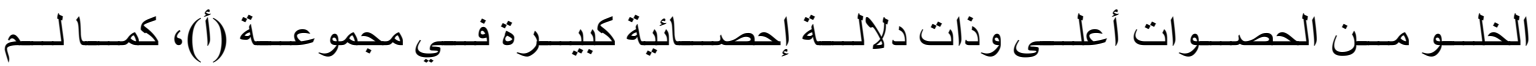

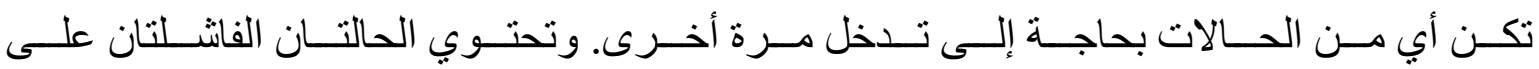

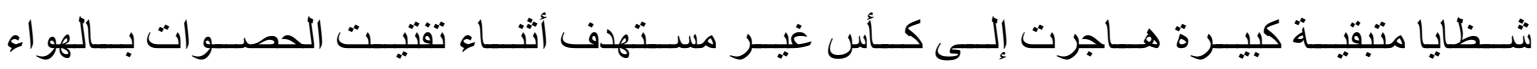

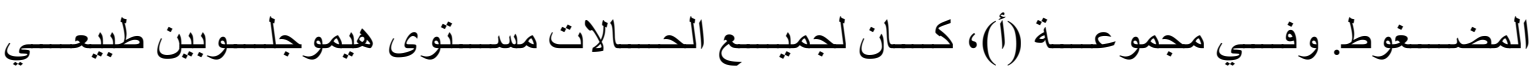

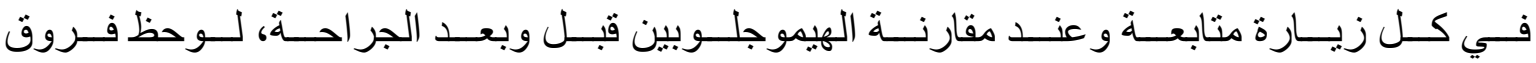
ذات دلالة إحصائية كبيرة بين مستوى الهيموجلوبين قبل وبعد الجراحة.

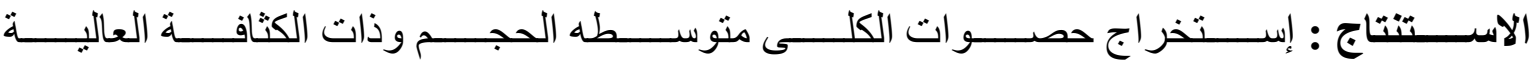

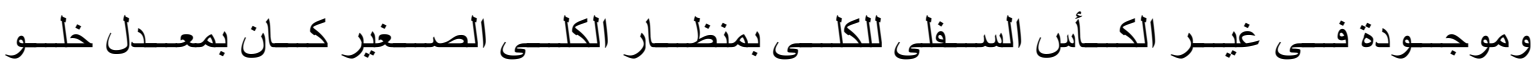

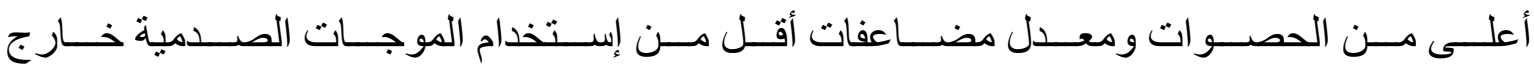
الجسم.

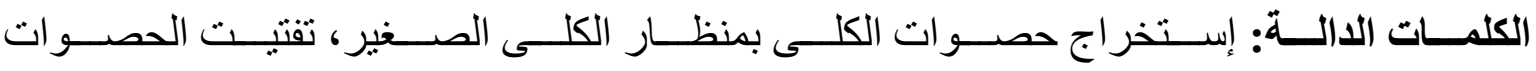

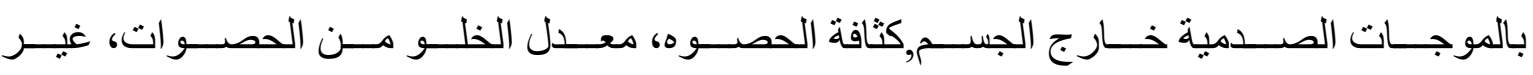
الكأس السفلى، حصو ات الكلى. 